\title{
Comparison of two approaches for the direct optimisation of a car engine intake port
}

\author{
J.J. Maisonneuve ${ }^{1, a}$, F. Pécot ${ }^{1}$, A. Pagès ${ }^{1}$, M. Albertelli ${ }^{1}$ and J. Visconti ${ }^{1}$ \\ 1 Sirehna, 1, rue de la Noë, BP 42105, 44321 Nantes Cedex 3, France \\ 2 Technocentre Renault, 1 avenue du Golf, 78288 Guyancourt, France
}

Received 14 June 2007, accepted 21 January 2008

\begin{abstract}
The shape optimisation involving complex flows, and based on direct CFD approaches, has now become very possible within industrial contexts, thanks to recent advances in optimal design technologies. However, one key remaining issue is related with the way the shape deformations are modelled. This paper presents the development of such an optimisation procedure, applied to a car engine air intake port, and compares two alternative methods for the shape deformation modelling. Global port variations are defined through a set of shape parameters. The first option consists in building a CAD model (Catia v5) to allow deformations, followed by a grid generation stage with tools enabling to generate and adapt the mesh to shape variations (Gridgen). The second option consists in starting from a grid done for a given shape, and defining parametric deformations on this grid, with a morphing tool (Sculptor). Then, after both modelling methods, the flow calculation is carried out with a CFD solver (Fluent). The resulting fields are processed by a flow post-processor (Fieldview) and by a Matlab procedure to extract the global criteria involved as optimisation objectives: the flow rate and the tumble. All this process is bundled within the modeFRONTIER multi-objective design environment. Similar optimisation approaches were led on both cases, involving mixed response surfaces - direct calculation evaluations of the designs, and a multi-objective genetic algorithm. The paper presents the main results, highlights the advantages and shortcomings of both modelling methods, and provides guidelines for their use and improvements, in view of practical industrial applications.
\end{abstract}

\section{Introduction}

Thanks to advances in CFD and in optimal design methods and tools, the optimisation of complex flows has now become manageable within an industrial context. Among the remaining difficulties to expand this approach, parametric modelling of complex shapes, and its influence on the optimisation results, is investigated here.

The paper focuses on the optimisation of a car engine air intake port and presents the results obtained with two optimisation chains based on two alternative methods for the shape parametric deformation: CAD based deformation and grid morphing.

\section{CAD based optimisation}

A parametric CAD model of the geometry was built using Catia v5 [1] (see Fig. 1). Four parameters were selected to define global port variations: the ogive height, the angle

\footnotetext{
${ }^{a}$ Corresponding authors: jean-jacques .maisonneuve@sirehna.com, maryan.sidorkiewicz@renault.com
}

between the base and the ogive, the angle between the ogive and the intake, and the joining radius between the two intake arms.

Then, a grid auto adapting to the CAD geometric variations was built using the Gridgen grid generator [2]. The central part of this grid is made up of tetrahedral cells while the plenum chamber and the outlet volume are made up of prism layers obtained by extrusion (see Fig. 1). The final grid contains about 90000 cells (for half a shape).

CFD calculations were performed with the NavierStokes solver Fluent [3] and the results were postprocessed by a flow post-processor (Fieldview [4]) and by a Matlab [5] procedure to extract the global criteria involved as optimisation conflicting objectives: the mass flow rate and the tumble.

All this tools were embedded into the multi-objective design environment modeFRONTIER [6].

A multi-objective genetic algorithm (MOGA II) was used for the calculations [7]. This robust algorithm has a spread wise behaviour and enables a wide exploration of the design space, from an initial population of designs. But it needs a large number of calculation points to 


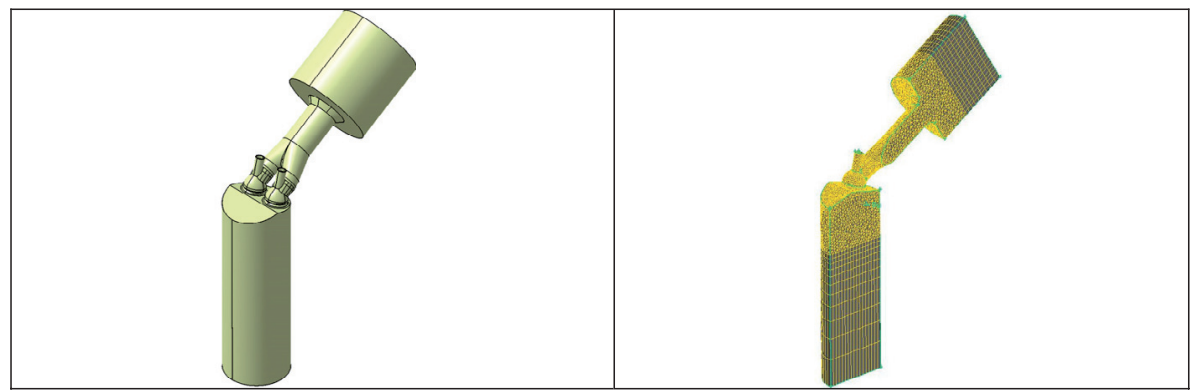

Fig. 1. Parametric CAD model and grid associated.

converge to the pareto front, which can be critical when the calculation time of each design is large, as in the present case. To solve this issue, a mixed approach has been retained: mixing real calculation of the designs $(20 \%$ of the evaluations) and virtual calculations based on response surfaces $(80 \%)$, built within modeFRONTIER and based on Gaussian Processes. The optimisation was led until convergence to a Pareto front enabling a complete understanding of the design problem.

Figure 2 shows the results obtained in terms of tumble and flow rate.

From the previous parameters and their associated range of variations, the best results for both objectives are: mass flow rate $+6.9 \%$ (variant A) and tumble $+24.7 \%$ (variant $\mathrm{C}$ ). Figure 3 shows the velocity results for the initial design and the variants $\mathrm{A}$ and $\mathrm{C}$.

This procedure provides most of the useful information for the designer to improve the shape and fulfil objectives. However, the freedom in shape is limited by the ability of the CAD tools to tolerate some combinations of parameters. This is illustrated by the difference between real and virtual Pareto designs.

\section{Parametric grid based optimisation}

An alternative method for the shape deformation modelling, is the definition of parametric deformations on the grid. Starting from a grid of a given shape, deformations have been defined with a morphing tool (Sculptor [8]). Figure 4 shows examples of grid deformations.

The rest of the calculation chain (flow calculation and post-processing) is similar to the previous one. The input variables and the range of variations are, as far as possible, identical to those of the CAD based chain.

The optimisation strategy is also similar to the previous one. The results are shown in Figure 5.

The best results for both objectives are: mass flow rate $+3.7 \%$ (variant $\mathrm{A}$ ) and tumble $+18.7 \%$ (variant $\mathrm{C}$ ). The figure hereafter shows the velocity results for the initial design and the variants $\mathrm{A}$ and $\mathrm{C}$.

This procedure also provides valuable design information. In addition, it is more robust in terms of ability to tolerate deformations, even if some issues may occur in some case (control of some local deformations, mesh quality in local areas). This can be show in the Pareto front, where real and virtual calculations match well.

\section{Conclusions}

Two optimisation chains based on two different approaches for shape parametric modelling have been implemented on an industrial test case, with off the shelf CAE tools. The whole was driven by the modeFRONTIER environment, which supports lack of reliability associated with shape deformations and flow calculations, thanks to robust multi-objective algorithms (as genetic algorithm) and calculation management process. Large computing times associated with CFD calculations have been managed through an hybrid approach mixing real calculations and response surfaces.

Both methods have led to similar global information regarding the design trends, the dependence of performances on shape variables, the directions of improvement. This information is not developed here.

The applicability of these approaches is demonstrated, within an industrial context where the optimisation problem is often posed as "find the best possible solutions with the people and tools available and within the allocated time for design" rather than as "find the best overall possible solution". The order of magnitude of manpower needed for such a study is now 2 to 3 weeks, depending on the complexity of the shape and objectives. The overall elapsed time is mainly depending on the computing resources, size of grids used to solve the flow problem, and number of shape parameters and objectives. In the present case, the calculation time was about $1.5 \mathrm{~h}$ for one point, on a moderately powerful mono-processor platform $(2.6 \mathrm{GHz})$, which led to a global calculation time of about five days. This can be highly improved with current up to date multi-processor platforms.

As regards the compared performance of both shape deformation approaches, the following conclusions can be extracted from the exercise.

The shape modification by direct action on the CAD parameters of the geometry is possible, but:

- This approach requires an strong modelling effort, in which the designer has to conciliate the constraints imposed by the CAD tool, with those related to the desired geometrical deformations. This may lead for complex shapes in huge costs associated with shape modelling. 


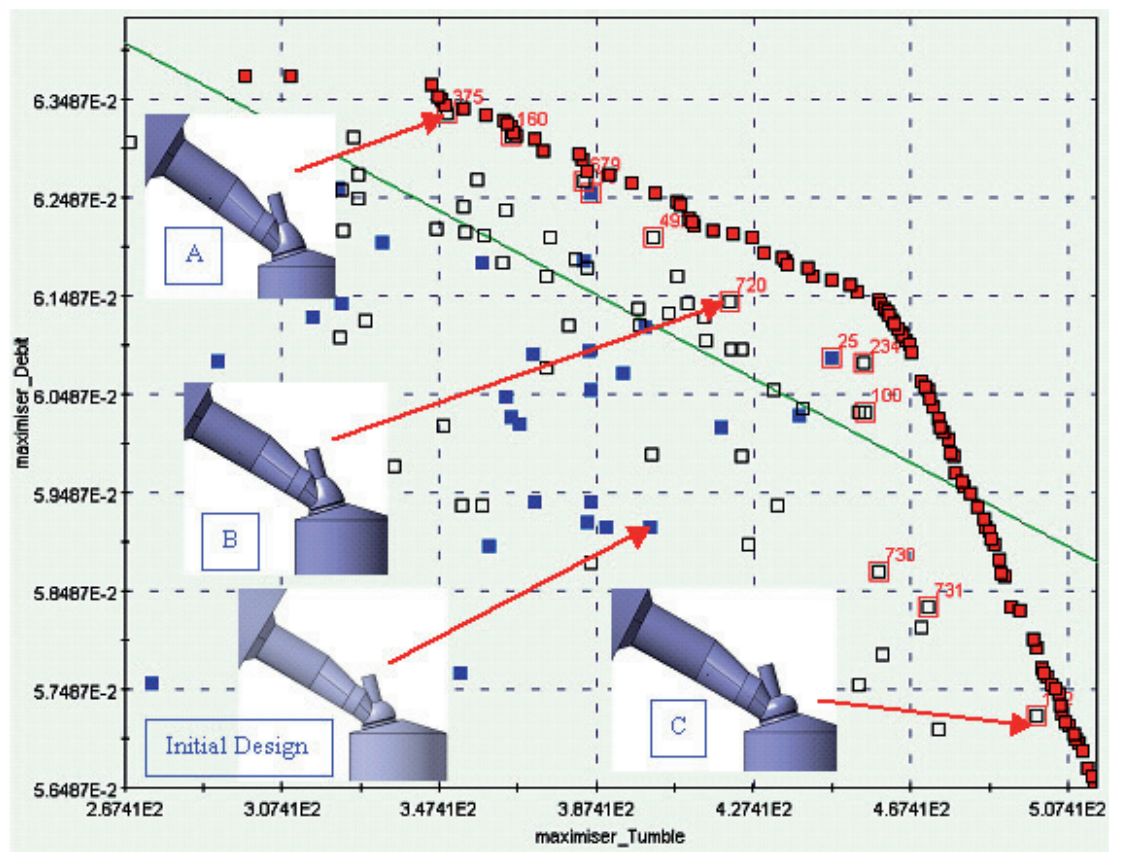

Fig. 2. Tumble vs. flow rate - DOE (blue), real calculations (white), real (numbers) and virtual (red) pareto set.

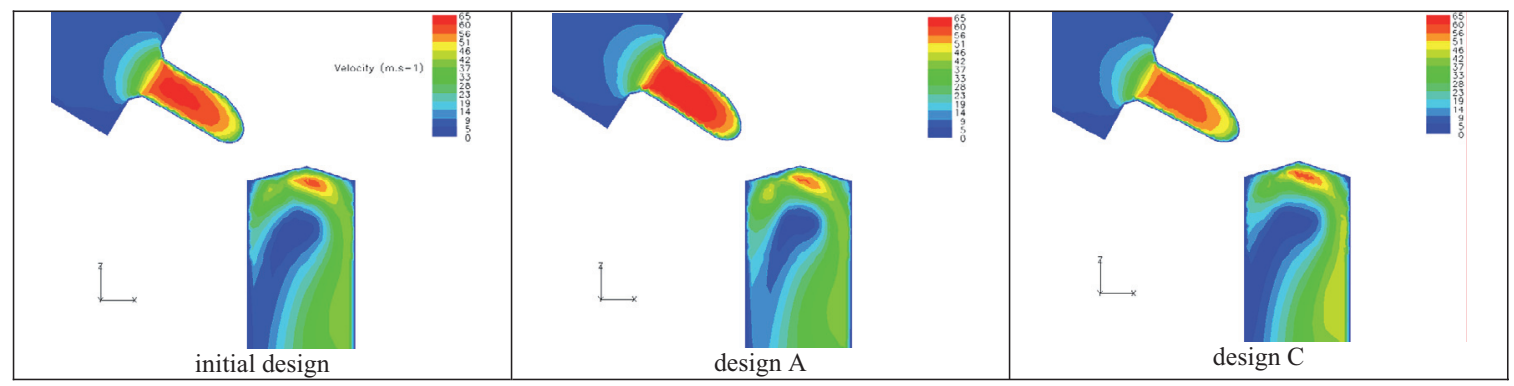

Fig. 3. Velocity results for some designs.

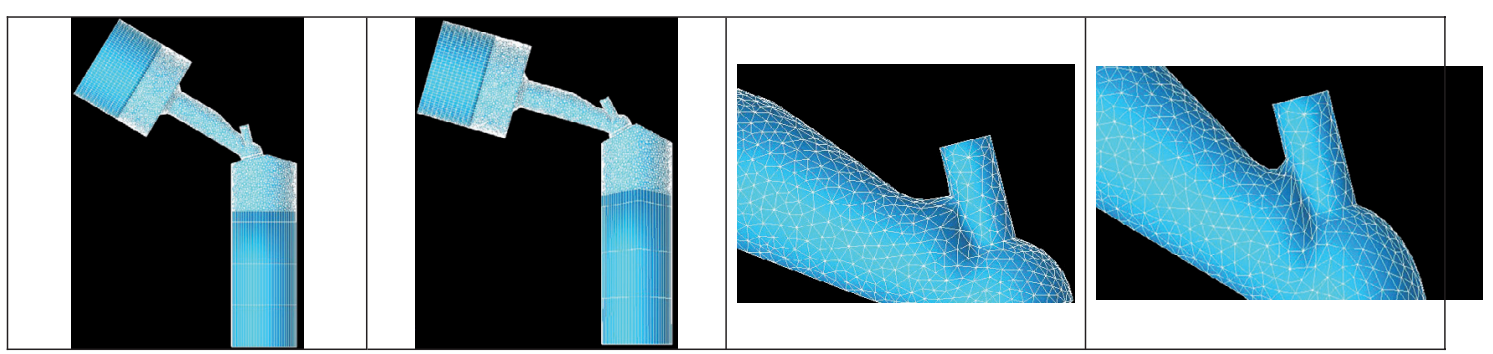

Fig. 4. Examples of grid deformations with Sculptor.

- In addition, such a modelling necessarily leads to some limitations in term of feasibility of the geometry: in the present case, $30 \%$ of the tentative designs failed, especially at the beginning of the optimisation. This figure can rise up significantly in case of complex shape.

- To compensate this shortcoming, it is recommended to have a preliminary analysis of the CAD generation robustness, and to adjust of variables and their range of variation, prior to the any optimisation. This investigation can be done within the optimisation environment.
- It is also necessary to have optimisation environments and algorithms compatible with this lack of reliability of the CAD shape generation.

- In addition, such a modelling requires, for the CFD application, to automatically create a mesh adapted to each shape. This also constitutes an great difficulty for complex geometry, and some failures related to the generation of the mesh can also be noticed.

- This can be treated with sophisticated meshing tools fitted with command language capabilities, which allow to adapt the mesh to the CAD geometrical 


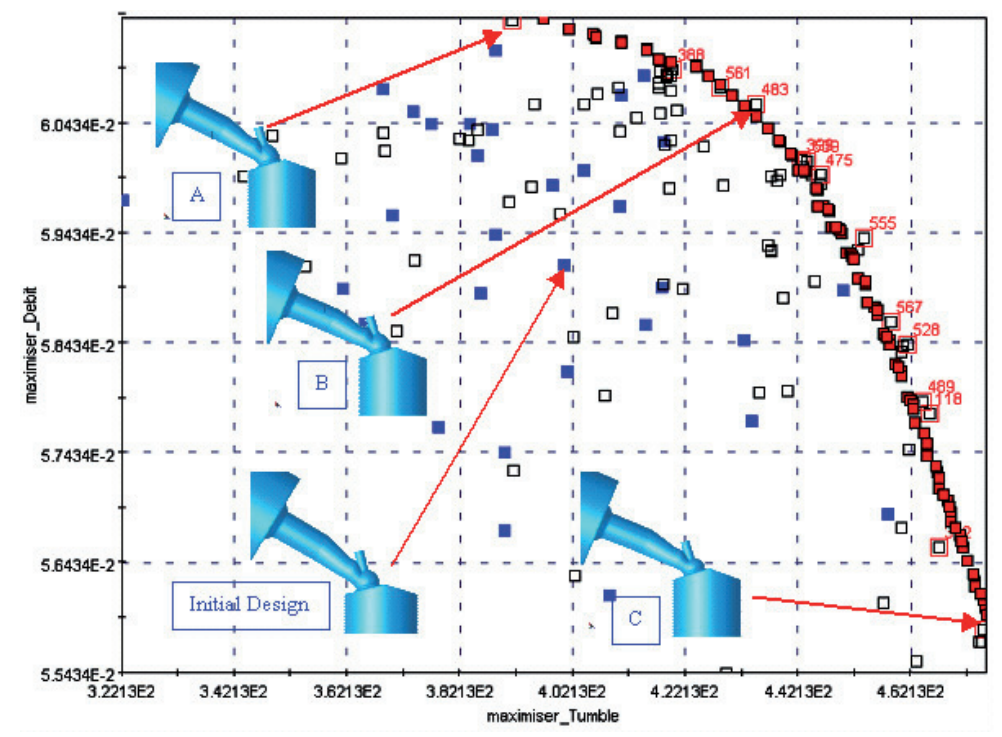

Fig. 5. Tumble vs flow rate - DOE (blue), real calculations (white), real (numbers) and virtual (red) pareto set.

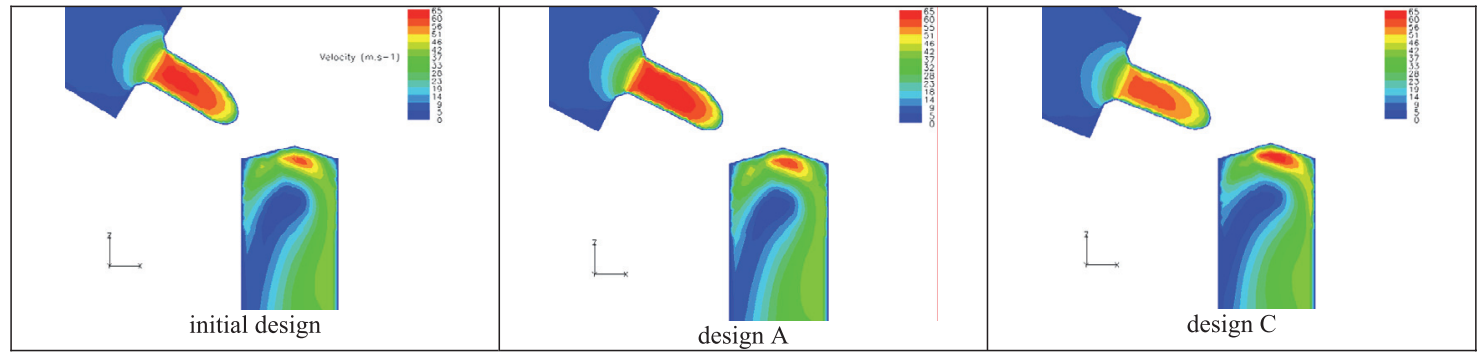

Fig. 6. Velocity results for some designs.

variations. This remains nevertheless expensive in grid modelling time.

- This can also be dealt with directly in the calculation chain, where several types of grid can be generated, adapted to CAD topology in entry, according for example to the values of design parameters.

- The mesh definition is also associated to a grid type and size. Any modification of this induces a redefinition of the grid process.

- Furthermore, if the relevant solutions founded by the optimisation process are close to the unfeasible CAD domain, the pareto front can be significantly affected.

- It is then necessary to detect this, in order to understand this behaviour, and if necessary to modify the model, to authorise more relevant variations.

An approach based on grid deformations allows to avoid some of these problems:

- No auto-adaptive mesh generation is needed: an initial manual mesh is enough to implement the method.

- Deformations can exactly be applied at the locations desired by the user, without any of the contingencies related to a CAD tool.

- Deformations remain valid whatever the grid used, which allows a modification of grid without redefinition of parameterisation.
- The deformation modelling time remains small (compared to the creation of the CAD model).

- There are few restrictions in terms of parameter variations: all the combinations of parameter values lead to a deformation of the grid, which have nevertheless to be investigated before validating the space of variation.

- However, some restrictions concerning the mesh quality can exist, for some ranges or amplitudes of deformations. This must be dealt with by using feasibility or grid quality criteria to reject these cases. In practice, the number of such non-calculable cases is definitely less important than for the CAD approach, and this robustness facilitates the optimisation.

- Some deformations can also be difficult to model with this approach, especially when the desired variations are large and combined.

- Another shortcoming of this approach is related to the fact that the optimisation result is a mesh of the geometry. To exploit the result in terms of CAD, it is necessary to reimport the result into the CAD tool, through various means, but with loss of the parametric information.

So the decision to choose one or the other approach must be considered against the above conclusions, depending on the particular case and design environment. 
This work was performed within the OMD project, supported by the French "Agence Nationale de la Recherche", in the scope of the 2005 RNTL programme. The test case and associated data were provided by RENAULT.

\section{References}

1. CATIA is a CAD tool edited by Dassault Systèmes, http://www.3ds.com

2. GRIDGEN is a grid generator edited by Pointwise Inc., http://www.pointwise.com/

3. FLUENT, is a CFD solver edited by ANSYS Inc., http:// www.fluent.com/
4. FIELDVIEW is a flow post-processor edited by Intelligent Light Inc, http://www.ilight.com/

5. MATLAB is edited by The Mathworks, http://www.mathworks.com/

6. modeFRONTIER is a multi-objective optimisation design environment edited by ESTECO,

http://www.esteco.com/

7. MOGA-II, An improved Multi-Objective Genetic Algorithm, Technical Report 2003-006, Silvia Poles, ESTECO, December 2003

8. SCULPTOR is a grid shape deformation tool edited by Optimal Solutions Software, http://www.optimalsolutions.us/ 